\title{
FoveaBox: Beyound Anchor-Based Object Detection
}

\author{
Tao Kong ${ }^{\circledR}$, Fuchun Sun, Fellow, IEEE, Huaping Liu, Yuning Jiang, Lei Li, and Jianbo Shi
}

\begin{abstract}
We present FoveaBox, an accurate, flexible, and completely anchor-free framework for object detection. While almost all state-of-the-art object detectors utilize predefined anchors to enumerate possible locations, scales and aspect ratios for the search of the objects, their performance and generalization ability are also limited to the design of anchors. Instead, FoveaBox directly learns the object existing possibility and the bounding box coordinates without anchor reference. This is achieved by: (a) predicting category-sensitive semantic maps for the object existing possibility, and (b) producing category-agnostic bounding box for each position that potentially contains an object. The scales of target boxes are naturally associated with feature pyramid representations. In FoveaBox, an instance is assigned to adjacent feature levels to make the model more accurate.We demonstrate its effectiveness on standard benchmarks and report extensive experimental analysis. Without bells and whistles, FoveaBox achieves state-of-the-art single model performance on the standard COCO and Pascal VOC object detection benchmark. More importantly, FoveaBox avoids all computation and hyper-parameters related to anchor boxes, which are often sensitive to the final detection performance. We believe the simple and effective approach will serve as a solid baseline and help ease future research for object detection. The code has been made publicly available at https:/github.com/taokong/FoveaBox.
\end{abstract}

Index Terms-Object detection, anchor free, foveabox.

\section{INTRODUCTION}

$\mathbf{O}$ BJECT detection requires the solution of two main tasks: recognition and localization. Given an arbitrary image, an object detection system needs to determine whether there are any instances of semantic objects from predefined categories and, if present, to return the spatial location and extent. To add the localization functionality to generic object detection systems, sliding window approaches have been the method of choice for many years [1], [2].

Recently, deep learning techniques have emerged as powerful methods for learning feature representations automatically from data [3]-[5]. For object detection, the anchor-based

Manuscript received December 29, 2019; revised May 6, 2020 and May 26, 2020; accepted June 5, 2020. Date of publication June 23, 2020; date of current version July 13, 2020. This work was supported in part by the National Science Foundation of China under Grant 61621136008, Grant 91848206, and Grant U1613212 and in part by the German Research Foundation under Grant DFG TRR-169. The associate editor coordinating the review of this manuscript and approving it for publication was Dr. Jiantao Zhou. (Corresponding author: Fuchun Sun.)

Tao Kong, Yuning Jiang, and Lei Li are with the ByteDance AI Lab, Beijing 100098, China (e-mail: taokongcn@ gmail.com).

Fuchun Sun and Huaping Liu are with the Department of Computer Science and Technology, Tsinghua University, Beijing National Research Center for Information Science and Technology (BNRist), Beijing 100084, China (e-mail: fcsun@tsinghua.edu.cn).

Jianbo Shi is with the GRASP Laboratory, University of Pennsylvania, Philadelphia, PA 19104 USA.

Digital Object Identifier 10.1109/TIP.2020.3002345

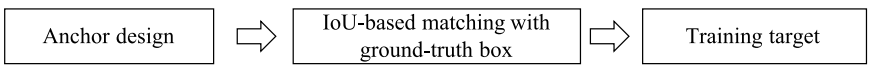

Fig. 1. The anchor-based object detection frameworks need to (a) design anchors according to the ground-truth box distributions; (b) match anchors with ground-truth boxes to generate training target (anchor classification and refinement); and (c) utilize the target generated by (b) for training.

Region Proposal Networks [6] are widely used to serve as a common component for searching possible regions of interest for modern object detection frameworks [7]-[9]. In short, anchor method suggests dividing the box space into discrete bins and refining the object box in the corresponding bin. Most state-of-the-art detectors rely on anchors to enumerate the possible locations, scales, and aspect ratios for target objects [10]. Anchors are regression references and classification candidates to predict proposals for two-stage detectors or final bounding boxes for single-stage detectors. Nevertheless, anchors can be regarded as a feature-sharing sliding window scheme to cover the possible locations of objects.

However, anchors must be carefully designed and used in object detection frameworks. (a) One of the most important factors in designing anchors is how densely it covers the instance location space. To achieve a good recall rate, anchors are carefully designed based on the statistics computed from the training/validation set [9], [11]. (b) One design choice based on a particular dataset is not always applicable to other applications, which harms the generality [12]. (c) At training phase, anchor-methods rely on the intersection-overunion (IoU) to define the positive/negative samples, which introduces additional computation and hyper-parameters for an object detection system [13].

In contrast, our human vision system can recognize the instance in space and predict the boundary given the visual cortex map, without any pre-defined shape template [14]. In other words, we human naturally recognize the object in the visual scene without enumerating the candidate boxes. Inspired by this, an intuitive question to ask is, is the anchor scheme the optimal way to guide the search of objects? And further, could we design an accurate object detection framework without anchors or candidate boxes? Without anchors, one may expect a complex method is required to achieve comparable performance. However, we show that a surprisingly simple and flexible system can match, even surpass the prior stateof-the-art object detection results without any requirement of candidate boxes.

To this end, we present FoveaBox, a completely anchor-free framework for object detection. FoveaBox is motivated from the fovea of human eyes: the center of the vision field is with 

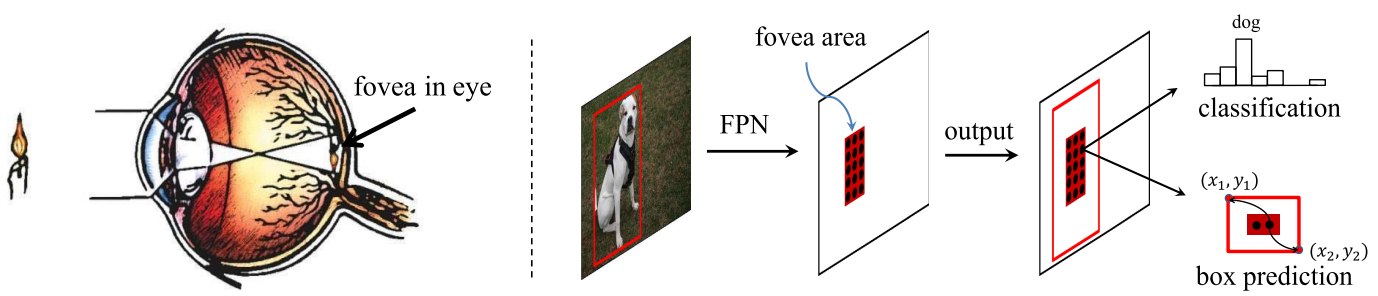

Fig. 2. FoveaBox object detector. For each output spacial position that potentially presents an object, FoveaBox directly predicts the confidences for all target categories and the bounding box.

the highest visual acuity (Fig.2 left), which is necessary for activities where visual detail is of primary importance [15]. FoveaBox jointly predicts the locations where the object's center area is likely to exist as well as the bounding box at each valid location. In FoveaBox, each target object is predicted by category scores at center area, associated with 4-d bounding box, as shown in Fig. 2 right. At training phase, we do not need to utilize anchors, or IoU matching to generate training target. Instead, the training target is directly generated by ground-truth boxes.

In the literature, some works attempted to leverage the FCNs-based framework for object detection such as DenseBox [16]. However, to handle the bounding boxes with different sizes, DenseBox [16] crops and resizes training images to a fixed scale. Thus DenseBox has to perform detection on image pyramids, which is against FCN's philosophy of computing all convolutions once. Besides, more significantly, these methods are mainly used in special domain objection detection such as scene text detection [17], [18] and face detection [19]. In constract, our FoveaBox can process could process more than 10 images on much challenging generic object detection (eg., 80 classes in MS COCO [20]).

To demonstrate the effectiveness of the proposed detection scheme, we combine the recent progress of feature pyramid networks [21] and our detection head to form the framework of FoveaBox. Without bells and whistles, FoveaBox gets stateof-the-art single-model results on the $\mathrm{COCO}$ object detection task. Compared with the anchor-based RetinaNet, FoveaBox gets 2.2 AP gains, which also surpasses most of previously published anchor based single-model results. We believe the simple training/inference manner of FoveaBox, together with the flexibility and accuracy, will benefit future research on object detection and relevant topics.

\section{RELATED WORK}

Object detection aims to localize and recognize every object instance with a bounding box [10]. The DPM [22] and its variants [23] have been the dominating methods for years. These methods use image descriptors such as HOG [24], SIFT [25], and LBP [26] as features and sweep through the entire image to find regions with a class-specific maximum response. With the great success of the deep learning on large scale object recognition [4], [27], [28], several works based on CNN have been proposed [29]-[32]. In this section, we mainly discuss the relations and differences of our FoveaBox and some previous works in details.

\section{A. Anchor-Based Object Detection}

The anchor-based object detection frameworks can be generally grouped into two factions: two-stage, proposal driven detectors and one-stage, proposal free methods.

1) Two-Stage Object Detection: The emergence of Faster R-CNN [6] establishes the dominant position of two-stage anchor-based detectors. Faster R-CNN consists of a region proposal network (RPN) and a region-wise prediction network (R-CNN) [29], [33] to detect objects. After that, lots of algorithms are proposed to improve its performance, including architecture redesign and reform [21], [34], [35], context and attention mechanism [36], training strategy and loss function [37], [38], feature fusion and enhancement [39]-[41]. Anchors are regression references and classification candidates to predict proposals for two-stage detectors.

2) One-Stage Object Detection: One-stage anchor-based detectors have attracted much attention because of their high computational efficiency. SSD [7] spreads out anchor boxes on multi-scale layers within a ConvNet to directly predict object category and anchor box offsets. Thereafter, plenty of works are presented to boost its performance in different aspects, such as fusing context information from different layers [42]-[44], training from scratch [45], introducing new loss function [9], anchor refinement and matching [46], [47], feature enrichment and alignment [48]-[51]. Anchors serve as the references boxes for final setections for one-stage detectors.

\section{B. Anchor-Free Explorations}

The most popular anchor-free detector might be YOLOv1 [52]. The YOLOv1 system takes a $448 \times 448$ image as input, and outputs $7 \times 7$ grid cells for box prediction. Since only one point is used to predict bounding box, YOLOv1 suffers from low recall [11]. As a result, both YOLOv2 [11] and YOLOv3 [53] utilize anchors to ensure high recall. DenseBox [16] and Unitbox [19] also did not utilize anchors for detection. The family of detectors have been considered unsuitable for generic object detection due to difficulty in multi-scale object detection and the recall being relatively low [54]. To detect object of multi-scales, DenseBox must utilize image pyramid, which needs several seconds to process one image. Our FoveaBox could process more than 10 images per second with better performance. Guided-Anchoring [13] predicts the scales and aspect ratios centered at the corresponding 
locations. It still relies on predefined anchors to optimize the object shape, and utilizes the center points to give the best predictions. In contrast, FoveaBox predicts the (left, top, right, bottom) boundaries of the object for each foreground position.

RepPoints [55] proposes to represent objects as a set of sample points, and utilizes deformable convolution [56] to get more accurate features. FSAF [57] predicts the best feature level to train each instance with anchor-free paradigm. We propose to assign objects to multiple adjacent pyramid levels for more robust prediction. FCOS [54] tries to solve object detection in a per-pixel prediction fashion. It relies on centerness map for suppressing low-quality detections. Instead, FoveaBox directly predict the final class probability without centerness voting, which is more simple. The CenterNet [58] represent each instance by its features at the center point. In FoveaBox, we utilize the fovea area controlled by $\sigma$ to predict the objects, which is more flexible. We note that FoveaBox, CenterNet [58] and FCOS [54] are concurrent works. Also, there are several works trying to extend the anchor-free concept into instance segmentation [59], [60].

Another family of anchor-free methods follow the 'bottomup' way [61], [62]. In CornerNet [61], the authors propose to detect an object bounding box as a pair of key-points, the top-left corner and the bottom-right corner. CornerNet adopts the Associative Embedding [63] technique to separate different instances. Also there are some following works in bottom-up grouping manner [64], [65]. CenterNet [62] tries to improve the precision and recall of CornerNet by cascade corner pooling and center pooling. It should be noted that the bottom-up methods also do not need anchors during training and inference. Compared with 'bottom-up' methods, our FoveaBox does not need any embedding or grouping techniques at post-processing stage.

\section{FoveaBox}

FoveaBox is conceptually simple: It contains a backbone network and a fovea head network. The backbone is responsible for computing a convolutional feature map over an entire input image and is an off-the-shelf convolutional network. The fovea head is composed of two sub-branches, the first branch performs per pixel classification on the backbone's output; the second branch performs box prediction for each position that potentially covered by an object.

\section{A. Review of FPN and Anchors}

We begin by briefly reviewing the Feature Pyramid Network (FPN) used for object detection [21]. In general, FPN uses a top-down architecture with lateral connections to build an in-network feature pyramid from a single-scale input. FPN is independent of a specific task. For object detection, each level of the pyramid in FPN is used for detecting objects at a specific scale. On each feature pyramid, anchor-based methods uniformly place $A$ anchors on each of the $H \times W$ spacial position. After computing the IoU overlap between all anchors and the ground-truth boxes, the anchor-based methods can define training targets. Finally, the pyramid features are utilized to optimize the targets.
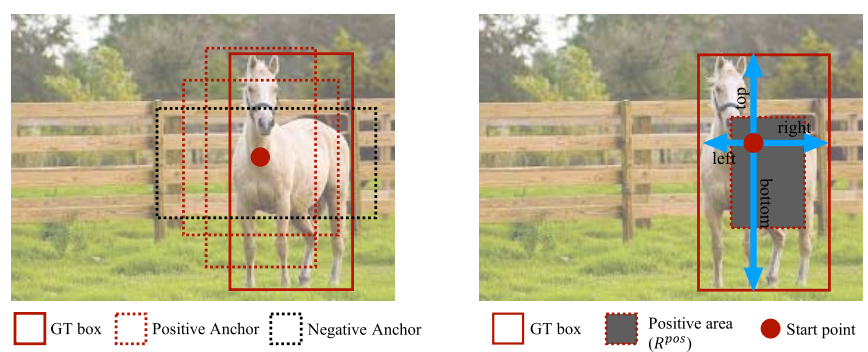

Fig. 3. Anchor-based object detection v.s. FoveaBox object detection. left: The anchor-based method uniformly places $A$ ( $A=3$ in this example) anchors on each output spacial position, and utilizes IoU to define the positive/negative anchors; right: FoveaBox directly defines positive/negative samples for each output spacial position by ground-truth boxes, and predicts the box boundaries from the corresponding position.

\section{B. FoveaBox}

FoveaBox directly predicts the object existing possibility and the corresponding boundary for each position potential contained by an instance. In this section, we introduce the key components step-by-step.

1) Object Occurrence Possibility: Given a valid ground-truth box denoted as $\left(x_{1}, y_{1}, x_{2}, y_{2}\right)$. We first map the box into the target feature pyramid $P_{l}$

$$
\begin{aligned}
& x_{1}^{\prime}=\frac{x_{1}}{s_{l}}, \quad y_{1}^{\prime}=\frac{y_{1}}{s_{l}}, \quad x_{2}^{\prime}=\frac{x_{2}}{s_{l}}, \quad y_{2}^{\prime}=\frac{y_{2}}{s_{l}}, \\
& c_{x}^{\prime}=0.5\left(x_{2}^{\prime}+x_{1}^{\prime}\right), \quad c_{y}^{\prime}=0.5\left(y_{2}^{\prime}+y_{1}^{\prime}\right), \\
& w^{\prime}=x_{2}^{\prime}-x_{1}^{\prime}, \quad h^{\prime}=y_{2}^{\prime}-y_{1}^{\prime},
\end{aligned}
$$

where $s_{l}$ is the down-sample factor. The positive area $R^{\text {pos }}$ on the score map is designed to be roughly a shrunk version of the original one (Fig. 3 right):

$$
\begin{aligned}
& x_{1}^{\text {pos }}=c_{x}^{\prime}-0.5 \sigma w^{\prime}, \quad y_{1}^{\text {pos }}=c_{y}^{\prime}-0.5 \sigma h^{\prime}, \\
& x_{2}^{\text {pos }}=c_{x}^{\prime}+0.5 \sigma w^{\prime}, \quad y_{2}^{\text {pos }}=c_{y}^{\prime}+0.5 \sigma h^{\prime},
\end{aligned}
$$

where $\sigma$ is the shrunk factor. At training phase, each cell inside the positive area is annotated with the corresponding target class label. The negative area is the whole feature map excluding area in $R^{\text {pos }}$. For predicting, each output set of pyramidal heat-map has $C$ channels, where $C$ is the number of categories, and is of size $H \times W$. Each channel is a binary mask indicating the possibility for a class, like FCNs in semantic segmentation [69]. The positive area usually accounts for a small portion of the whole feature map, so we adopt Focal Loss [9] to train this branch.

2) Scale Assignment: While our goal is to predict the boundary of the target objects, directly predicting these numbers is not stable, due to the large scale variations of the objects. Instead, we divide the scales of objects into several bins, according to the number of feature pyramidal levels. Each pyramid has a basic scale $r_{l}$ ranging from 32 to 512 on pyramid levels $P_{3}$ to $P_{7}$, respectively. The valid scale range of the target boxes for pyramid level $l$ is computed as

$$
\left[r_{l} / \eta, r_{l} \cdot \eta\right],
$$

where $\eta$ is set empirically to control the scale range for each pyramid. Target objects not in the corresponding scale 


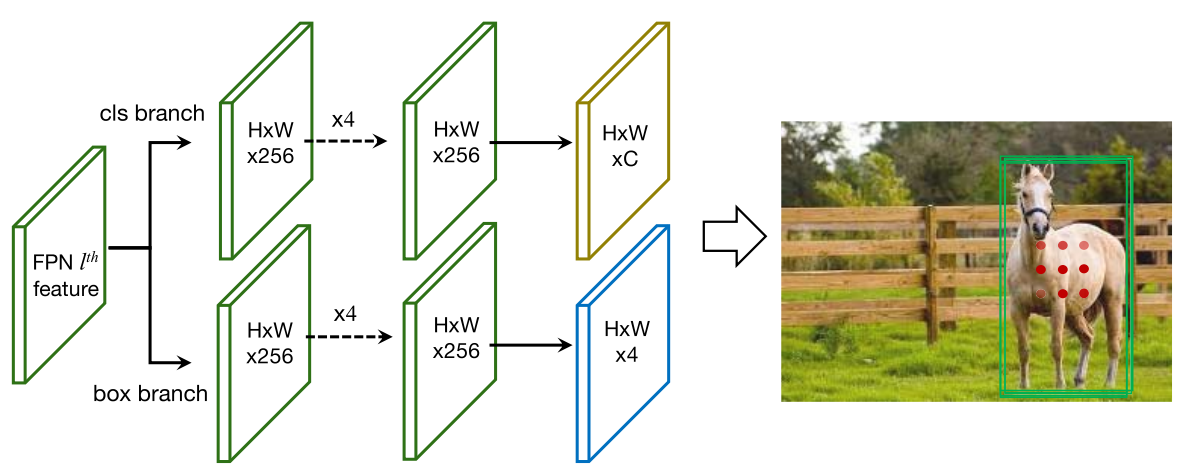

Fig. 4. On each FPN feature level, FoveaBox attaches two subnetworks, one for classifying the corresponding cells and one for predict the $\left(x_{1}, y_{1}, x_{2}, y_{2}\right)$ of ground-truth object box. Right is the score output map with their corresponding predicted boxes before feeding into non-maximum suppression (NMS). The score probability in each position is denoted by the color density. More examples are shown in Fig.5.
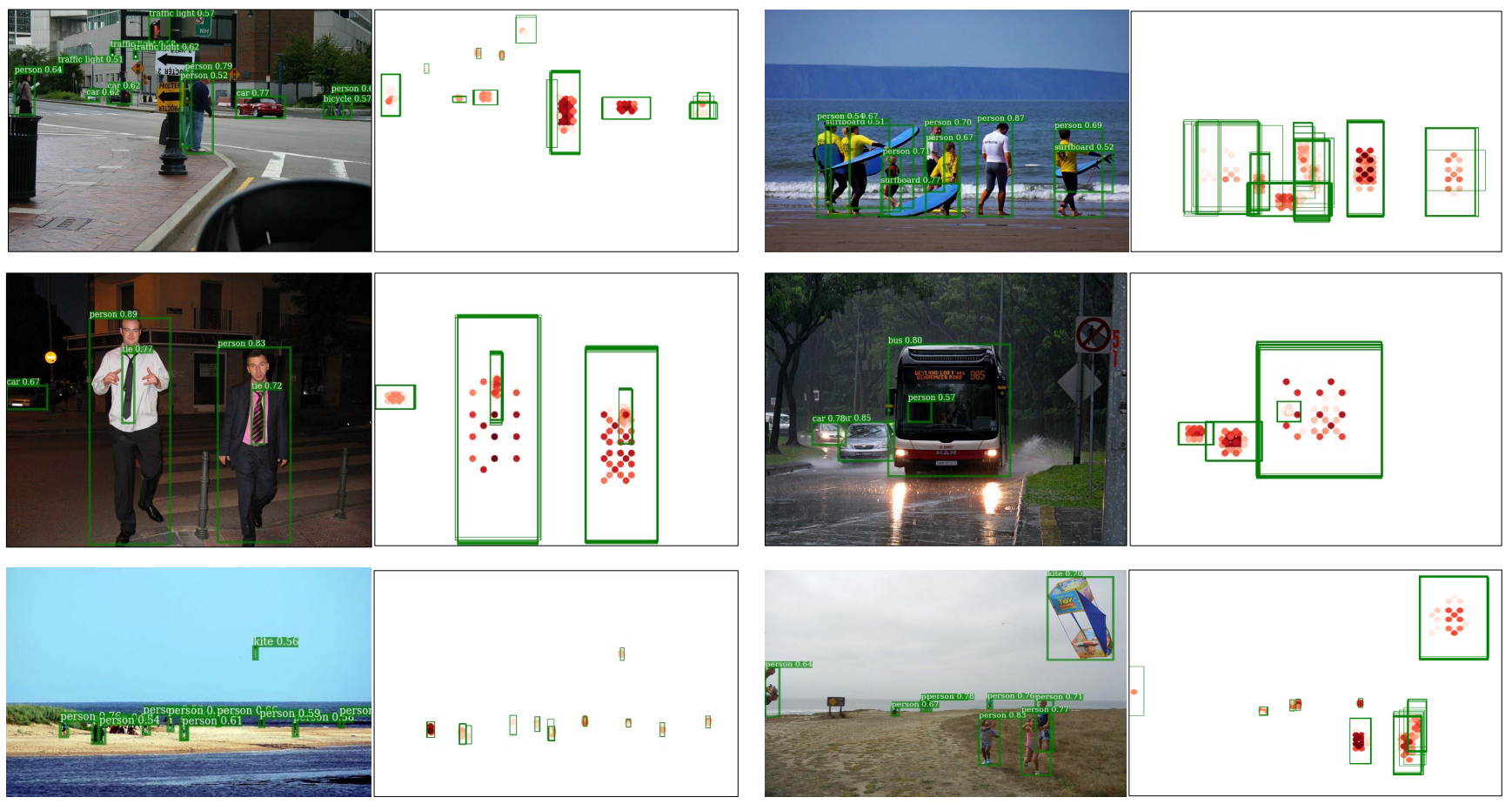

Fig. 5. FoveaBox results on the COCO minival set. These results are based on ResNet-101, achieving a single model box AP of 38.6. For each pair, left is the detection results with bounding box, category, and confidence. Right is the score output map with their corresponding bounding boxes before feeding into non-maximum suppression (NMS). The score probability in each position is denoted by the color density.

range are ignored during training. Note that an object may be detected by multiple pyramids of the networks, which is different from previous practice that maps objects to only one feature pyramid [8].

Assigning objects to multiple adjacent pyramid levels has two advantages: a) The adjacent pyramid levels usually have similar semantic capacity, so FoveaBox could optimize these pyramid features simultaneously. b) The training sample number for each pyramid has largely enlarged, which make the training process more stable. In the experiment section, we show that our scale assignment strategy gives 3.1 AP gains compared with single level training and predicting.

3) Box Prediction: Each ground-truth bounding box is specified in the way $G=\left(x_{1}, y_{1}, x_{2}, y_{2}\right)$. Starting from a positive point $(x, y)$ in $R^{p o s}$, FoveaBox directly computes the normalized offset between $(x, y)$ and four boundaries:

$$
\begin{aligned}
& t_{x_{1}}=\log \frac{s_{l}(x+0.5)-x_{1}}{r_{l}}, \\
& t_{y_{1}}=\log \frac{s_{l}(y+0.5)-y_{1}}{r_{l}}, \\
& t_{x_{2}}=\log \frac{x_{2}-s_{l}(x+0.5)}{r_{l}}, \\
& t_{y_{2}}=\log \frac{y_{2}-s_{l}(y+0.5)}{r_{l}} .
\end{aligned}
$$

This function first maps the coordinate $(x, y)$ to the input image, then computes the normalized offset between the projected coordinate and $G$. Finally the targets are regularized with the $\log$-space function. $r_{l}$ is the basic scale defined in section III-B.2. 
TABLE I

Object Detection Single-Model Results v.s. State-of-the-Arts on COCO test-dev. We Show Results for Our FoveaBox Models With 800 Input SCale. FoveaBoX-Align Indicates Utilizing Feature Alignment Discussed

\begin{tabular}{l|c|cccccc} 
& backbone & $\mathrm{AP}$ & $\mathrm{AP}_{50}$ & $\mathrm{AP}_{75}$ & $\mathrm{AP}_{S}$ & $\mathrm{AP}_{M}$ & $\mathrm{AP}_{L}$ \\
\hline two-stage methods & & & & & & & \\
Faster R-CNN w FPN [21] & ResNet-101 & 36.2 & 59.1 & 39.0 & 18.2 & 39.0 & 48.2 \\
Mask R-CNN [8] & ResNet-101 & 38.2 & 60.3 & 41.7 & 20.1 & 41.1 & 50.2 \\
Faster R-CNN by G-RMI [63] & Inception-ResNet-v2 & 34.7 & 55.5 & 36.7 & 13.5 & 38.1 & 52.0 \\
Faster R-CNN w TDM [64] & Inception-ResNet-v2 & 36.8 & 57.7 & 39.2 & 16.2 & 39.8 & 52.1 \\
Relation Network [65] & DCN-101 & 39.0 & 58.6 & 42.9 & - & - & - \\
IoU-Net [38] & ResNet-101 & 40.6 & 59.0 & - & - & - & - \\
Cascade R-CNN [47] & ResNet-101 & 42.8 & 62.1 & 46.3 & 23.7 & 45.5 & 55.2 \\
\hline one-stage methods & & & & & & & \\
YOLOv2 [51] & Darknet-19 & 21.6 & 44.0 & 19.2 & 5.0 & 22.4 & 35.5 \\
YOLOv3 [52] & Darknet-53 & 33.0 & 57.9 & 34.4 & 18.3 & 35.4 & 41.9 \\
SSD513 [41] & ResNet-101 & 31.2 & 50.4 & 33.3 & 10.2 & 34.5 & 49.8 \\
DSSD513 [41] & ResNet-101 & 33.2 & 53.3 & 35.2 & 13.0 & 35.4 & 51.1 \\
RetinaNet [9] & ResNet-101 & 39.1 & 59.1 & 42.3 & 21.8 & 42.7 & 50.2 \\
RetinaNet [9] & ResNeXt-101 & 40.8 & 61.1 & 44.1 & 24.1 & 44.2 & 51.2 \\
RPDet [54] & ResNeXt-101 & 41.0 & 62.9 & 44.3 & 23.6 & 44.1 & 51.7 \\
FCOS [53] & ResNeXt-101 & 42.1 & 62.1 & 45.2 & 25.6 & 44.9 & 52.0 \\
CornerNet [58] & Hourglass-104 & 40.5 & 56.5 & 43.1 & 19.4 & 42.7 & 53.9 \\
ExtremeNet [61] & Hourglass-104 & 40.1 & 55.3 & 43.2 & 20.3 & 43.2 & 53.1 \\
CenterNet [57] & Hourglass-104 & 42.1 & 61.1 & 45.9 & 24.1 & 45.5 & 52.8 \\
\hline ours & & & & & & & \\
FoveaBox & ResNet-101 & 40.8 & 61.4 & 44.0 & 24.1 & 45.3 & 53.2 \\
FoveaBox & ResNeXt-101 & 42.3 & 62.9 & 45.4 & 25.3 & 46.8 & 55.0 \\
FoveaBox-align & ResNet-101 & 42.1 & 62.7 & 45.5 & 25.2 & 46.6 & 54.5 \\
FoveaBox-align & ResNeXt-101 & $\mathbf{4 3 . 9}$ & $\mathbf{6 3 . 5}$ & $\mathbf{4 7 . 7}$ & $\mathbf{2 6 . 8}$ & $\mathbf{4 6 . 9}$ & $\mathbf{5 5 . 6}$
\end{tabular}

For simplicity, we adopt the widely used Smooth $L_{1}$ loss [6] to train the box prediction $L_{b o x}$. After targets being optimized, we can generate the box boundary for each cell $(x, y)$ on the output feature maps. ${ }^{1}$ In box branch, each output set of pyramidal heatmap has 4 channels, for jointly prediction of $\left(t_{x_{1}}, t_{y_{1}}, t_{x_{2}}, t_{y_{2}}\right)$.

4) Network Architecture: To demonstrate the generality of our approach, we instantiate FoveaBox with multiple architectures. For clarity, we differentiate between: (i) the convolutional backbone architecture used for feature extraction over an entire image, and (ii) the network head for computing the final results. Most of the experiments are based on the head architecture as shown in Fig.4. We also utilize different head variants to further study the generality. More complex designs have the potential to improve performance but are not the focus of this work.

5) Implementations: We adopt the widely used FPN networks for fair comparison. Concretely, we construct a pyramid with levels $\left\{P_{l}\right\}, l=3,4, \cdots, 7$, where $l$ indicates pyramid level. $P_{l}$ has $1 / 2^{l}$ resolution of the input. All pyramid levels have $C=256$ channels. Fovea head is attached on each pyramid level. Parameters are shared across all pyramid levels.

FoveaBox is trained with stochastic gradient descent (SGD). We use synchronized SGD over 4 GPUs with a total of 16 images per minibatch (4 images per GPU). Unless otherwise specified, all models are trained for 12 epochs with an initial learning rate of 0.01 , which is then divided by 10 at 8th and again at 11th epochs. Weight decay of 0.0001 and momentum of 0.9 are used. Only standard horizontal image flipping is used for data augmentation. During inference, we first use a confidence threshold of 0.05 to filter out predictions with low confidence. Then, we select the top

\footnotetext{
${ }^{1}$ Eq.(4) and its inverse transformation can be easily implemented by an element-wise layer in modern deep learning frameworks [70], [71].
}

1000 scoring boxes from each prediction layer. Next, NMS with threshold 0.5 is applied for each class separately. Finally, the top-100 scoring predictions are selected for each image. Although there are more intelligent ways to perform postprocessing, such as bbox voting [72], Soft-NMS [73] or test-time image augmentations, in order to keep simplicity and to fairly compare against the baseline models, we do not use those tricks here.

Two-stage detectors rely on region-wise sub-networks to further classify the sparse region proposals. Since FoveaBox could also generate region proposals by changing the model head to class agnostic scheme, we believe it could further improve the performance of two-stage detectors, which beyond the focus of this paper.

\section{EXPERIMENTS}

We present experimental results on the bounding box detection track of the MS COCO and Pascal VOC benchmarks. For COCO, all models are trained on trainval35k. If not specified, ResNet-50-FPN backbone and a 600 pixel train and test image scale are used to do the ablation study. We report lesion and sensitivity studies by evaluating on the minival split. For our main results, we report COCO AP on the test-dev split, which has no public labels and requires use of the evaluation server. For Pascal VOC, all models are trained on trainval2007 and trainval2012, and evaluated on test2 007 subset, following common practice [6], [7].

\section{A. Main Results}

We compare FoveaBox to the state-of-the-art methods in Table I. All instantiations of our model outperform baseline variants of previous state-of-the-art models. The first group of detectors on Table I are two-stage detectors, the second group one-stage detectors, and the last group the FoveaBox 
TABLE II

VARYING ANCHOR DENSITY AND FOVEABoX

\begin{tabular}{c|cc|ccc} 
method & \#sc & \#ar & $\mathrm{AP}$ & $\mathrm{AP}_{50}$ & $\mathrm{AP}_{75}$ \\
\hline RetinaNet & 1 & 1 & 30.2 & 49.0 & 31.7 \\
RetinaNet & 2 & 1 & 31.9 & 50.0 & 34.1 \\
RetinaNet & 3 & 1 & 31.9 & 49.4 & 33.8 \\
RetinaNet & 2 & 3 & 34.2 & 53.1 & 36.5 \\
RetinaNet & 3 & 3 & 34.2 & 53.2 & 36.9 \\
RetinaNet & 4 & 3 & 33.9 & 52.1 & 36.2 \\
\hline FoveaBox & - & - & $\mathbf{3 5 . 1}$ & $\mathbf{5 4 . 3}$ & $\mathbf{3 7 . 1}$
\end{tabular}

detector. FoveaBox outperforms all single-stage detectors under ResNet-101 backbone, under all evaluation metrics. This includes the recent one-stage CornerNet and ExtremeNet [61], [64]. FoveaBox also outperforms most of two-stage detectors, including FPN [21], Mask R-CNN [8] and IoU-Net [38].

\section{B. Ablation Study}

1) Qualitative Results: Fig.5 shows the detection outputs of FoveaBox. Points and boxes with class probability larger than 0.5 are shown (before feeding into NMS). For each object, though there are several active points, the predicted boxes are very close to the ground-truth. These figures demonstrate that FoveaBox could directly generate accurate, robust box predictions, without the requirement of candidate anchors.

2) Various Anchor Densities and FoveaBox: One of the most important design factors in an anchor-based detection system is how densely it covers the space of possible objects. As anchor-based detectors use a fixed sampling grid, a popular approach for achieving high coverage of boxes is to use multiple anchors at each spatial position. One may expect that we can always get better performance when attaching denser anchors on each position. To verify this assumption, we sweep over the number of scale and aspect ratio anchors used at each spatial position and each pyramid level in RetinaNet, including a single square anchor at each location to 12 anchors per location (Table II). Increasing beyond 6-9 anchors does not show further gains. The saturation of performance w.r.t. density implies the handcrafted, over-density anchors do not offer an advantage.

Over-density anchors not only increase the foreground-background optimization difficulty, but also likely to cause the ambiguous position definition problem. For each output spatial location, there are $A$ anchors whose labels are defined by the IoU with the ground-truth. Among them, some of the anchors are defined as positive samples, while others are negatives. However they are sharing the same input features. The classifier needs to not only distinguish the samples from different positions, but also different anchors at the same position.

In contrast, FoveaBox explicitly predicts one target at each position and gets no worse performance than the best anchor-based model. Compare with the anchor based scheme, FoveaBox enjoys several advantages. (a) Since we only predict one target at each position, the output space has been reduced to $1 / A$ of the anchor-based method. (b) There is no ambiguous problem and the optimization target is more straightforward. (c) FoveaBox has fewer hyper-parameters,
TABLE III

Detection With DifFerent Aspect Ratios

\begin{tabular}{c|cccc} 
method & $\mathrm{AP}$ & $\mathrm{AP}_{u<3}$ & $\mathrm{AP}_{3<u<5}$ & $\mathrm{AP}_{u>5}$ \\
\hline RetinaNet & 34.2 & 36.5 & 24.5 & 10.2 \\
\hline FoveaBox & $\mathbf{3 5 . 1}$ & $\mathbf{3 6 . 8}$ & $\mathbf{2 6 . 8}$ & $\mathbf{1 6 . 4}$
\end{tabular}

TABLE IV

Region Propos al Performance

\begin{tabular}{c|c|ccc} 
method & backbone & $\mathrm{AR}_{100}$ & $\mathrm{AR}_{300}$ & $\mathrm{AR}_{1000}$ \\
\hline RPN & ResNet-50 & 44.5 & 51.1 & 56.6 \\
FoveaBox & ResNet-50 & $\mathbf{5 2 . 9}$ & $\mathbf{5 7 . 3}$ & $\mathbf{6 1 . 5}$
\end{tabular}
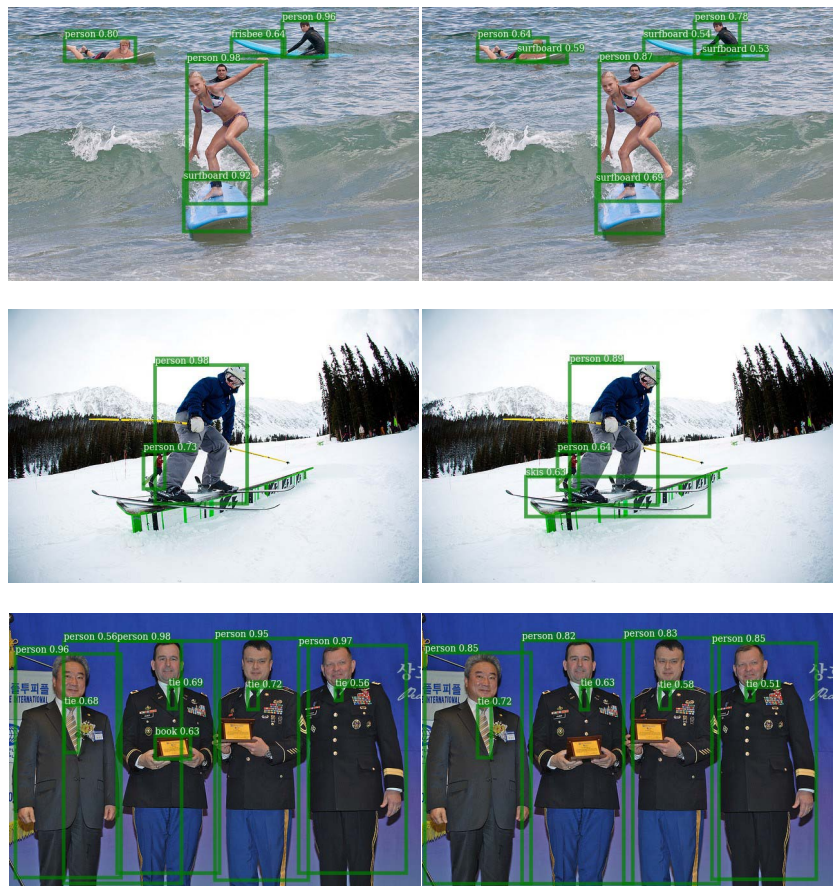

(a)

(b)

Fig. 6. Qualitative comparison of RetinaNet (a) and FoveaBox (b). Our model shows improvements in classes with large aspect ratios.

and is more flexible, since we do not need to extensively design anchors to see a relatively better choice.

3) FoveaBox Is More Robust to Box Distribution: One of the major benefits of FoveaBox is the robust prediction of bounding boxes. To verify this, we divide the boxes in the validation set into three groups according to the ground-truth aspect ratios $u=\max \left(\frac{h}{w}, \frac{w}{h}\right)$. We compare FoveaBox and RetinaNet at different aspect ratio thresholds, as shown in Table III. We see that both methods get best performance when $u$ is low. Although FoveaBox also suffers performance decrease when $u$ increases, it is much better than the baseline model. Some qualitative examples are shown in Fig. 6.

4) Generating High-Quality Region Proposals: Changing the classification target to class-agnostic head is straightforward and could generate region proposals. We compare the proposal performance against FPN-based RPN [21] and evaluate average recalls (AR) with different numbers of proposals on minival set, as shown in Table IV. Surprisingly, our method outperforms the RPN baseline by a large margin, among all criteria. Specifically, with top 100 region proposals, 
TABLE V

Feature Alignment and Group Normalization (Resnet-50, 800 Scale)

\begin{tabular}{c|cc|ccc} 
cls branch & alignment & $\mathrm{GN}$ & $\mathrm{AP}$ & $\mathrm{AP}_{50}$ & $\mathrm{AP}_{75}$ \\
\hline $256(3 \times 3) \rightarrow 256(3 \times 3) \rightarrow 256(3 \times 3) \rightarrow 256(3 \times 3)$ & & & 36.4 & 56.2 & 38.7 \\
$256(3 \times 3) \rightarrow 256(3 \times 3) \rightarrow 256(3 \times 3) \rightarrow 256(3 \times 3)$ & $\checkmark$ & & 36.8 & 56.5 & 38.9 \\
$256(3 \times 3) \rightarrow 256(3 \times 3) \rightarrow 256(3 \times 3) \rightarrow 256(3 \times 3)$ & $\checkmark$ & $\checkmark$ & 37.1 & 56.7 & 39.2 \\
\hline $1024(3 \times 3) \rightarrow 1024(1 \times 1)$ & & & 36.7 & 57.0 & 39.1 \\
$1024(3 \times 3) \rightarrow 1024(1 \times 1)$ & $\checkmark$ & & 37.2 & 57.4 & 39.4 \\
$1024(3 \times 3) \rightarrow 1024(1 \times 1)$ & $\checkmark$ & $\checkmark$ & 37.5 & 58.2 & 39.5 \\
$1024(3 \times 3) \rightarrow 1024(1 \times 1), 2 \times$ epochs & $\checkmark$ & $\checkmark$ & 37.9 & 58.4 & 40.4 \\
$1024(3 \times 3) \rightarrow 1024(1 \times 1), 2 \times$ epochs, mstrain & $\checkmark$ & $\checkmark$ & 40.1 & 60.8 & 42.5
\end{tabular}

TABLE VI

DIFFERENT INPUT RESOLUTIONS AND MODELS

\begin{tabular}{c|ccc} 
net-depth-scale & $\mathrm{AP}$ & $\mathrm{AP}_{50}$ & $\mathrm{AP}_{75}$ \\
\hline FoveaBox-50-400 & $31.9+1.4$ & 49.6 & 33.8 \\
FoveaBox-50-600 & $35.1+0.9$ & 54.3 & 37.1 \\
FoveaBox-50-800 & $36.4+0.9$ & 56.2 & 38.7 \\
\hline FoveaBox-101-400 & $33.3+1.4$ & 51.0 & 35.0 \\
FoveaBox-101-600 & $37.0+1.0$ & 56.4 & 39.3 \\
FoveaBox-101-800 & $38.6+0.9$ & 58.0 & 41.2
\end{tabular}

TABLE VII

VARYING $\eta(\sigma=0.4)$

\begin{tabular}{c|ccc}
$\eta$ & $\mathrm{AP}$ & $\mathrm{AP}_{50}$ & $\mathrm{AP}_{75}$ \\
\hline 1.0 & 32.0 & 50.4 & 31.8 \\
1.5 & 34.1 & 53.3 & 36.0 \\
2.0 & $\mathbf{3 5 . 1}$ & $\mathbf{5 4 . 4}$ & $\mathbf{3 7 . 0}$ \\
2.5 & 35.0 & 54.2 & 36.8 \\
3.0 & 34.3 & 53.3 & 36.5 \\
4.0 & 32.8 & 51.0 & 34.5
\end{tabular}

FoveaBox gets 52.9 AR, outperforming RPN by 8.4 points. This validates that our model's capacity in generating high quality region proposals.

5) Across Model Depth and Scale: Table VI shows FoveaBox utilizing different backbone networks and input resolutions. The train/inference settings are exactly the same as the baseline method [9]. Under the same settings, FoveaBox consistently gets $0.9 \sim 1.4$ higher AP. When comparing the inference speed, we find that FoveaBox models are about $1.1 \sim 1.3$ times faster than the RetinaNet counterparts.

6) Analysis of $\eta$ and $\sigma$ : In Eq.(3), $\eta$ controls the scale assignment extent for each pyramid. As $\eta$ increases, each pyramid will response to more scales of objects. Table VII shows the impact of $\eta$ on the final detection performance. Another important hyper-parameter is the shrunk factor $\sigma$ which controls the positive/negative samples. Table VIII shows the model performance with respect to $\sigma$ changes. In this paper, $\sigma=0.4$ and $\eta=2$ are used in other experiments.

7) IoU-Based Assignment v.s. Fovea Area: Another choice of defining the positive/negative samples is firstly gets the predicted box from the box branch, and then assign the target labels based on the IoU between the predicted boxes and ground-truth boxes. As shown in Table IX, the shrunk version gets better performance $(+0.4 \mathrm{AP})$ than the IoU-based assignment process.

8) Better Head and Feature Alignment: The most recent works [55], [74] suggest to align the features in one-stage
TABLE VIII

VARYING $\sigma(\eta=2.0)$

\begin{tabular}{c|ccc}
$\sigma$ & $\mathrm{AP}$ & $\mathrm{AP}_{50}$ & $\mathrm{AP}_{75}$ \\
\hline 0.2 & 34.1 & 53.2 & 36.0 \\
0.3 & 34.8 & 54.0 & 36.7 \\
0.4 & $\mathbf{3 5 . 1}$ & $\mathbf{5 4 . 4}$ & $\mathbf{3 7 . 0}$ \\
0.5 & 34.8 & 53.9 & 36.6 \\
0.6 & 34.1 & 53.1 & 36.0 \\
0.7 & 33.3 & 52.5 & 34.9
\end{tabular}

TABLE IX

LAbel Assignment Strategy (ResNet-50, 800 SCALE)

\begin{tabular}{c|ccc} 
assign method & $\mathrm{AP}$ & $\mathrm{AP}_{50}$ & $\mathrm{AP}_{75}$ \\
\hline IoU $(0.5 / 0.4)$ & 35.6 & 54.7 & 37.7 \\
IoU $(0.6 / 0.5)$ & 35.9 & 54.7 & 38.4 \\
IoU $(0.5 / 0.5)$ & 36.0 & 54.9 & 38.4 \\
\hline Fovea $(\sigma=0.4)$ & 36.4 & 56.2 & 38.7
\end{tabular}

object detection frameworks with anchors. In FoveaBox, we adopt deformable convolution [56] based on the box offset learned by Eq.(4) to refine the classification branch. FoveaBox works well when adding such techniques. Specifically, when we change the classification branch to a heavier head, together with feature alignment and GN, FoveaBox gets 40.1 AP using ResNet-50 as backbone! This experiment demonstrates the generality of our approach to the network design (Table V). For feature alignment, we adopt a $3 \times 3$ deformable convolutional layer [56] to implement the transformation, as shown in Fig.7. The offset input to the deformable convolutional layer is $\left(\hat{t}_{x_{1}}, \hat{t}_{y_{1}}, \hat{t}_{x_{2}}, \hat{t}_{y_{2}}\right)$ of the bbox output.

We also compare the performance of front features of box branch to refine the final classification branch. Table $\mathrm{X}$ gives the performance and speed with respect to different layers of box branch. It shows that using the front features really improves the speed. However, using the last layer gives the best AP performance.

9) Per-Class Difference: Fig. 8 shows per-class AP difference of FoveaBox and RetinaNet. Both of them are with ResNet-50-FPN backbone and 800 input scale. The vertical axis shows $\mathrm{AP}_{\text {Fovea Box }}-\mathrm{AP}_{\text {RetinaNet }}$. FoveaBox shows improvement in most of the classes.

10) Speed: We evaluate the inference time of FoveaBox and other methods for speed comparison. The experiments are performed on a single Nvidia V100 GPU by averaging 10 runs. The speed field could remotely reflect the actual run time of a model due to the difference in implementations. 


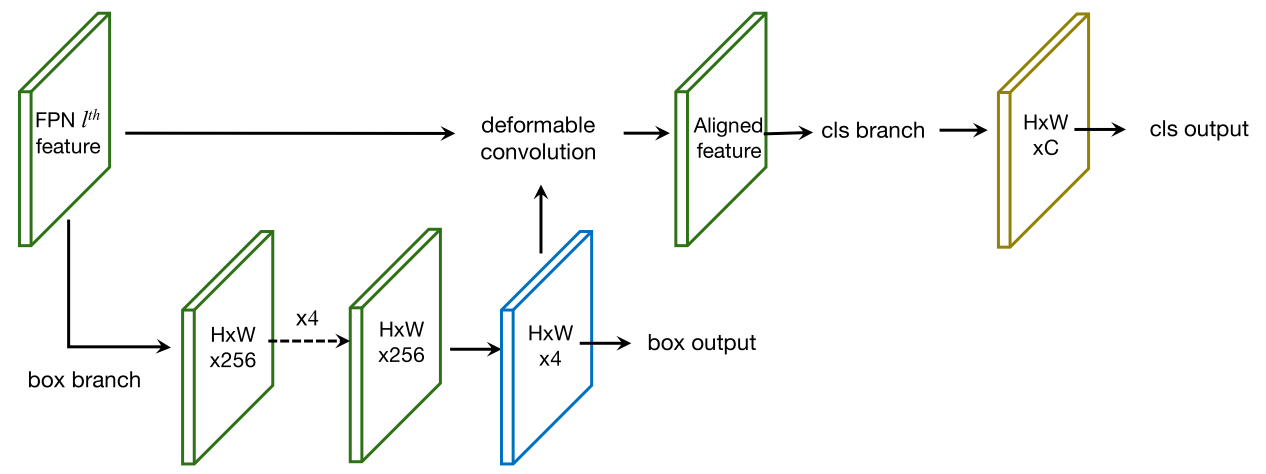

Fig. 7. Feature alignment process.

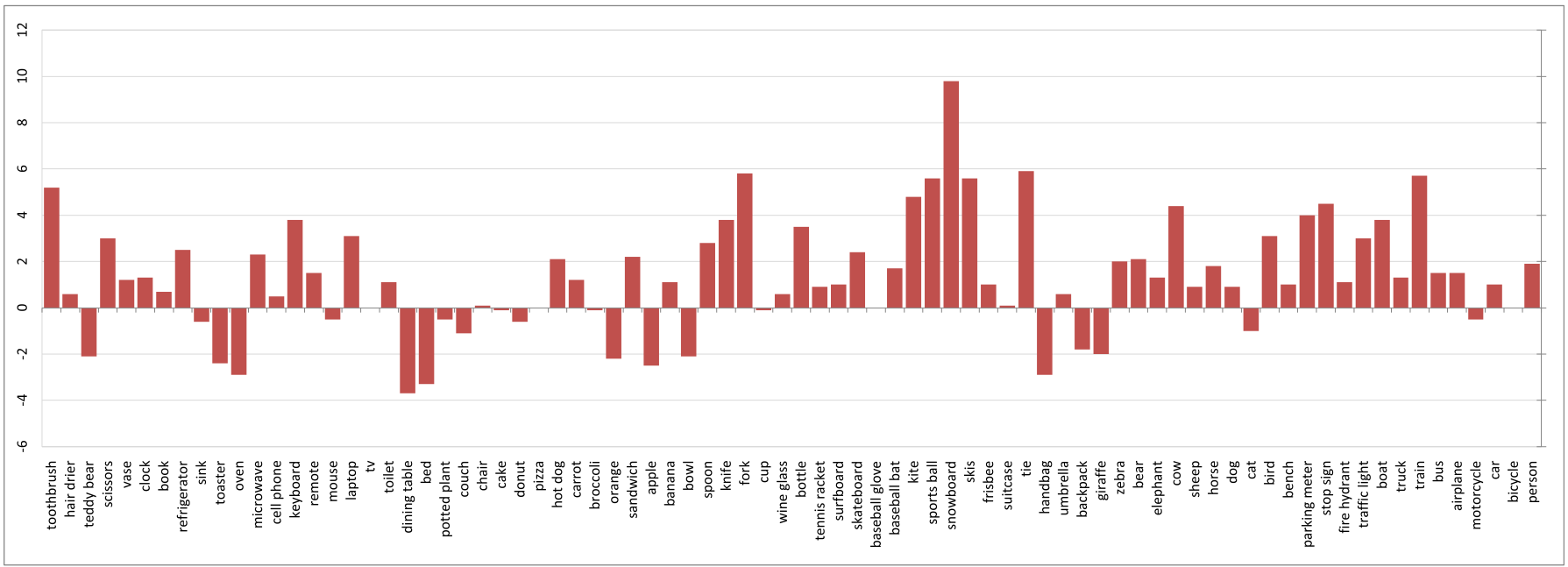

Fig. 8. AP difference of FoveaNet and RetinaNet on COCO dataset. Both models use ResNet-FPN-50 as backbone and 800 input scales.

TABLE $X$

Different Box Layer Numbers Used in FeAture Alignment

\begin{tabular}{c|ccc|c} 
layer number & $\mathrm{AP}$ & $\mathrm{AP}_{50}$ & $\mathrm{AP}_{75}$ & speed $(\mathrm{ms})$ \\
\hline 0 (w/ align) & 36.4 & 56.2 & 38.7 & 65 \\
1 & 36.7 & 56.3 & 39.9 & 67 \\
2 & 36.8 & 56.4 & 38.9 & 68 \\
3 & 36.9 & 56.6 & 39.0 & 70 \\
4 & 37.1 & 56.7 & 39.2 & 71
\end{tabular}

TABLE XI

SPEED COMPARISON

\begin{tabular}{c|c|c|c} 
method & backbone & AP & speed $(\mathrm{ms})$ \\
\hline CenterNet [57] & Hourglass-104 & 42.1 & 119 \\
ExtremNet [72] & Hourglass-104 & 40.1 & 301 \\
CornerNet [58] & Hourglass-104 & 40.5 & 291 \\
RetinaNet [9] & ResNeXt-101 & 40.8 & 106 \\
FCOS [53] & ResNeXt-101 & 42.1 & 98 \\
\hline FoveaBox & ResNeXt-101 & 42.3 & 95 \\
FoveaBox-align & ResNeXt-101 & 43.9 & 138
\end{tabular}

\section{Pascal VOC Dataset}

we conducted additional experiments on Pascal VOC object detection dataset [76]. This dataset covers 20 object categories, and the performance is measured by mean average precision $(\mathrm{mAP})$ at $\mathrm{IoU}=0.5$. All variants are trained on VOC2007 trainval and VOC2012 trainval, and tested on VOC2007 test dataset. Based on ResNet-50, we can compare the performances of FoveaBox and RetinaNet.
TABLE XII

FoveaBox on Pascal VOC Dataset

\begin{tabular}{c|c|c|c} 
method & backbone & mAP & speed $(\mathrm{ms})$ \\
\hline RetinaNet & ResNet-50-FPN & 75.5 & 74 \\
FoveaBox & ResNet-50-FPN & 76.6 & 61
\end{tabular}

\section{CONCLUSiON}

We have presented FoveaBox, a simple, effective, and completely anchor-free framework for generic object detection. By simultaneously predict the object position and the corresponding boundary, FoveaBox gives a clean solution for detecting objects without prior candidate boxes. We demonstrate its effectiveness on standard benchmarks and report extensive experimental analysis. We believe the simple and effective approach will serve as a solid baseline and help ease future research for object detection.

\section{REFERENCES}

[1] P. F. Felzenszwalb, R. B. Girshick, and D. McAllester, "Cascade object detection with deformable part models," in Proc. IEEE Comput. Soc. Conf. Comput. Vis. Pattern Recognit., Jun. 2010, pp. 2241-2248.

[2] C. H. Lampert, M. B. Blaschko, and T. Hofmann, "Beyond sliding windows: Object localization by efficient subwindow search," in Proc. IEEE Conf. Comput. Vis. Pattern Recognit., Jun. 2008, pp. 1-8. 
[3] K. Simonyan and A. Zisserman, "Very deep convolutional networks for large-scale image recognition," 2014, arXiv:1409.1556. [Online]. Available: http://arxiv.org/abs/1409.1556

[4] K. He, X. Zhang, S. Ren, and J. Sun, "Deep residual learning for image recognition," in Proc. IEEE Conf. Comput. Vis. Pattern Recognit. (CVPR), Jun. 2016, pp. 770-778.

[5] G. Huang, Z. Liu, L. Van Der Maaten, and K. Q. Weinberger, "Densely connected convolutional networks," in Proc. IEEE Conf. Comput. Vis. Pattern Recognit. (CVPR), Jul. 2017, pp. 4700-4708.

[6] S. Ren, K. He, R. Girshick, and J. Sun, "Faster R-CNN: Towards realtime object detection with region proposal networks," in Proc. Adv. Neural Inf. Process. Syst., 2015, pp. 91-99.

[7] W. Liu et al., "SSD: Single shot multibox detector," in Proc. Eur. Conf. Comput. Vis. Cham, Switzerland: Springer, 2016, pp. 21-37.

[8] K. He, G. Gkioxari, P. Dollár, and R. Girshick, "Mask R-CNN," in Proc. IEEE Int. Conf. Comput. Vis. (ICCV), Oct. 2017, pp. 2980-2988.

[9] T.-Y. Lin, P. Goyal, R. Girshick, K. He, and P. Dollár, "Focal loss for dense object detection," IEEE Trans. Pattern Anal. Mach. Intell., vol. 42, no. 2 , pp. 318-327, Feb. 2020.

[10] L. Liu et al., "Deep learning for generic object detection: A survey," 2018, arXiv:1809.02165. [Online]. Available: http://arxiv.org/ abs/1809.02165

[11] J. Redmon and A. Farhadi, "YOLO9000: Better, faster, stronger," in Proc. IEEE Conf. Comput. Vis. Pattern Recognit., Jul. 2017, pp. $7263-7271$.

[12] T. Yang, X. Zhang, Z. Li, W. Zhang, and J. Sun, "Metaanchor: Learning to detect objects with customized anchors," in Proc. Adv. Neural Inf. Process. Syst., 2018, pp. 318-328.

[13] J. Wang, K. Chen, S. Yang, C. C. Loy, and D. Lin, "Region proposal by guided anchoring," in Proc. IEEE/CVF Conf. Comput. Vis. Pattern Recognit. (CVPR), Jun. 2019, pp. 2965-2974.

[14] M. F. Bear, B. W. Connors, and M. A. Paradiso, Neuroscience, vol. 2. Philadelphia, PA, USA: Lippincott Williams \& Wilkins, 2007.

[15] M. Iwasaki and H. Inomata, "Relation between superficial capillaries and foveal structures in the human retina," Investigative Ophthalmol. Vis. Sci., vol. 27, no. 12, pp. 1698-1705, 1986.

[16] L. Huang, Y. Yang, Y. Deng, and Y. Yu, "DenseBox: Unifying landmark localization with end to end object detection," 2015, arXiv:1509.04874. [Online]. Available: http://arxiv.org/abs/1509.04874

[17] X. Zhou et al., "EAST: An efficient and accurate scene text detector," in Proc. IEEE Conf. Comput. Vis. Pattern Recognit. (CVPR), Jul. 2017, pp. 5551-5560.

[18] Z. Zhang, C. Zhang, W. Shen, C. Yao, W. Liu, and X. Bai, "Multi-oriented text detection with fully convolutional networks," in Proc. IEEE Conf. Comput. Vis. Pattern Recognit. (CVPR), Jun. 2016, pp. 4159-4167.

[19] J. Yu, Y. Jiang, Z. Wang, Z. Cao, and T. Huang, "Unitbox: An advanced object detection network," in Proc. 24th ACM Int. Conf. Multimedia, 2016, pp. 516-520.

[20] T.-Y. Lin et al., "Microsoft coco: Common objects in context," in Proc. Eur. Conf. Comput. Vis. Cham, Switzerland: Springer, 2014, pp. 740-755.

[21] T.-Y. Lin, P. Dollar, R. Girshick, K. He, B. Hariharan, and S. Belongie, "Feature pyramid networks for object detection," in Proc. CVPR, vol. 1, 2017, pp. 2117-2125.

[22] P. F. Felzenszwalb, R. B. Girshick, D. McAllester, and D. Ramanan, "Object detection with discriminatively trained part-based models," IEEE Trans. Pattern Anal. Mach. Intell., vol. 32, no. 9, pp. 1627-1645, Sep. 2010.

[23] H. Azizpour and I. Laptev, "Object detection using strongly-supervised deformable part models," in Proc. Eur. Conf. Comput. Vis. Berlin, Germany: Springer, 2012, pp. 836-849.

[24] N. Dalal and B. Triggs, "Histograms of oriented gradients for human detection," in Proc. IEEE Comput. Soc. Conf. Comput. Vis. Pattern Recognit. (CVPR), Jun. 2005, pp. 886-893.

[25] D. G. Lowe, "Distinctive image features from scale-invariant keypoints," Int. J. Comput. Vis., vol. 60, no. 2, pp. 91-110, Nov. 2004.

[26] X. Wang, T. X. Han, and S. Yan, "An HOG-LBP human detector with partial occlusion handling," in Proc. IEEE 12th Int. Conf. Comput. Vis., Sep. 2009, pp. 32-39.

[27] A. Krizhevsky, I. Sutskever, and G. E. Hinton, "ImageNet classification with deep convolutional neural networks," in Proc. Adv. Neural Inf. Process. Syst., 2012, pp. 1097-1105.

[28] J. Han, X. Yao, G. Cheng, X. Feng, and D. Xu, "P-CNN: Part-based convolutional neural networks for fine-grained visual categorization," IEEE Trans. Pattern Anal. Mach. Intell., early access, Aug. 6, 2019, doi: 10.1109/TPAMI.2019.2933510.
[29] R. Girshick, J. Donahue, T. Darrell, and J. Malik, "Rich feature hierarchies for accurate object detection and semantic segmentation," in Proc. IEEE Conf. Comput. Vis. Pattern Recognit., Jun. 2014, pp. 580-587.

[30] G. Cheng, P. Zhou, and J. Han, "Learning rotation-invariant convolutional neural networks for object detection in VHR optical remote sensing images," IEEE Trans. Geosci. Remote Sens., vol. 54, no. 12, pp. 7405-7415, Dec. 2016.

[31] D. Zhang, D. Meng, and J. Han, "Co-saliency detection via a self-paced multiple-instance learning framework," IEEE Trans. Pattern Anal. Mach. Intell., vol. 39, no. 5, pp. 865-878, May 2017.

[32] P. Sermanet, D. Eigen, X. Zhang, M. Mathieu, R. Fergus, and Y. LeCun, "OverFeat: Integrated recognition, localization and detection using convolutional networks," 2013, arXiv:1312.6229. [Online]. Available: http://arxiv.org/abs/1312.6229

[33] R. Girshick, "Fast R-CNN," in Proc. IEEE Int. Conf. Comput. Vis. (ICCV), Dec. 2015, pp. 1440-1448.

[34] Y. Li, Y. Chen, N. Wang, and Z.-X. Zhang, "Scale-aware trident networks for object detection," in Proc. IEEE Int. Conf. Comput. Vis., Oct. 2019, pp. 6054-6063.

[35] M. Najibi, B. Singh, and L. S. Davis, "AutoFocus: Efficient multi-scale inference," 2018, arXiv:1812.01600. [Online]. Available: http://arxiv.org/abs/1812.01600

[36] S. Bell, C. L. Zitnick, K. Bala, and R. Girshick, "Inside-outside net: Detecting objects in context with skip pooling and recurrent neural networks," in Proc. IEEE Conf. Comput. Vis. Pattern Recognit. (CVPR), Jun. 2016, pp. 2874-2883.

[37] A. Shrivastava, A. Gupta, and R. Girshick, "Training region-based object detectors with online hard example mining," in Proc. IEEE Conf. Comput. Vis. Pattern Recognit. (CVPR), Jun. 2016, pp. 761-769.

[38] B. Jiang, R. Luo, J. Mao, T. Xiao, and Y. Jiang, "Acquisition of localization confidence for accurate object detection," in Proc. Eur. Conf. Comput. Vis., Munich, Germany, 2018, pp. 8-14.

[39] T. Kong, A. Yao, Y. Chen, and F. Sun, "HyperNet: Towards accurate region proposal generation and joint object detection," in Proc. IEEE Conf. Comput. Vis. Pattern Recognit., Jun. 2016, pp. 845-853.

[40] T. Kong, F. Sun, A. Yao, H. Liu, M. Lu, and Y. Chen, "RON: Reverse connection with objectness prior networks for object detection," in Proc. IEEE Conf. Comput. Vis. Pattern Recognit. (CVPR), vol. 1, Jul. 2017, p. 2.

[41] F. Sun, T. Kong, W. Huang, C. Tan, B. Fang, and H. Liu, "Feature pyramid reconfiguration with consistent loss for object detection," IEEE Trans. Image Process., vol. 28, no. 10, pp. 5041-5051, Oct. 2019.

[42] C.-Y. Fu, W. Liu, A. Ranga, A. Tyagi, and A. C. Berg, "DSSD: Deconvolutional single shot detector," 2017, arXiv:1701.06659. [Online]. Available: http://arxiv.org/abs/1701.06659

[43] T. Kong, F. Sun, C. Tan, H. Liu, and W. Huang, "Deep feature pyramid reconfiguration for object detection," in Proc. Eur. Conf. Comput. Vis. (ECCV), 2018, pp. 169-185.

[44] F. Sun, T. Kong, W. Huang, C. Tan, B. Fang, and H. Liu, "Feature pyramid reconfiguration with consistent loss for object detection," IEEE Trans. Image Process., vol. 28, no. 10, pp. 5041-5051, Oct. 2019.

[45] Z. Shen, Z. Liu, J. Li, Y.-G. Jiang, Y. Chen, and X. Xue, "DSOD: Learning deeply supervised object detectors from scratch," in Proc. IEEE Int. Conf. Comput. Vis., Oct. 2017, pp. 1919-1927.

[46] T. Kong, F. Sun, H. Liu, Y. Jiang, and J. Shi, "Consistent optimization for single-shot object detection," 2019, arXiv:1901.06563. [Online]. Available: http://arxiv.org/abs/1901.06563

[47] S. Zhang, L. Wen, X. Bian, Z. Lei, and S. Z. Li, "Single-shot refinement neural network for object detection," in Proc. IEEE/CVF Conf. Comput. Vis. Pattern Recognit., Jun. 2018, pp. 4203-4212.

[48] Z. Cai and N. Vasconcelos, "Cascade R-CNN: Delving into high quality object detection," in Proc. IEEE Conf. Comput. Vis. Pattern Recognit., Jun. 2018, pp. 6154-6162.

[49] X. Zhu, H. Hu, S. Lin, and J. Dai, "Deformable ConvNets v2: More deformable, better results," 2018, arXiv:1811.11168. [Online]. Available: http://arxiv.org/abs/1811.11168

[50] M. Tan, R. Pang, and Q. V. Le, "EfficientDet: Scalable and efficient object detection," 2019, arXiv:1911.09070. [Online]. Available: http://arxiv.org/abs/1911.09070

[51] J. Guo et al., "Hit-detector: Hierarchical trinity architecture search for object detection," 2020, arXiv:2003.11818. [Online]. Available: http://arxiv.org/abs/2003.11818 
[52] J. Redmon, S. Divvala, R. Girshick, and A. Farhadi, "You only look once: Unified, real-time object detection," in Proc. IEEE Conf. Comput. Vis. Pattern Recognit. (CVPR), Jun. 2016, pp. 779-788.

[53] J. Redmon and A. Farhadi, "YOLOv3: An incremental improvement," 2018, arXiv:1804.02767. [Online]. Available: http://arxiv.org/abs/1804.02767

[54] Z. Tian, C. Shen, H. Chen, and T. He, "FCOS: Fully convolutional onestage object detection," 2019, arXiv:1904.01355. [Online]. Available: http://arxiv.org/abs/1904.01355

[55] Z. Yang, S. Liu, H. Hu, L. Wang, and S. Lin, "RepPoints: Point set representation for object detection," 2019, arXiv:1904.11490. [Online]. Available: http://arxiv.org/abs/1904.11490

[56] J. Dai et al., "Deformable convolutional networks," in Proc. IEEE Int. Conf. Comput. Vis., Oct. 2017, pp. 764-773.

[57] C. Zhu, Y. He, and M. Savvides, "Feature selective anchor-free module for single-shot object detection," 2019, arXiv:1903.00621. [Online]. Available: http://arxiv.org/abs/1903.00621

[58] X. Zhou, D. Wang, and P. Krähenbühl, "Objects as points," 2019, arXiv:1904.07850. [Online]. Available: http://arxiv.org/abs/1904.07850

[59] X. Wang, T. Kong, C. Shen, Y. Jiang, and L. Li, "SOLO: Segmenting objects by locations," 2019, arXiv:1912.04488. [Online]. Available: http://arxiv.org/abs/1912.04488

[60] X. Wang, R. Zhang, T. Kong, L. Li, and C. Shen, "SOLOv2: Dynamic, faster and stronger," 2020, arXiv:2003.10152. [Online]. Available: http://arxiv.org/abs/2003.10152

[61] H. Law and J. Deng, "CornerNet: Detecting objects as paired keypoints," in Proc. Eur. Conf. Comput. Vis. (ECCV), vol. 6, 2018, pp. 734-750.

[62] K. Duan, S. Bai, L. Xie, H. Qi, Q. Huang, and Q. Tian, "CenterNet: Keypoint triplets for object detection," in Proc. IEEE Int. Conf. Comput. Vis., Oct. 2019, pp. 6569-6578.

[63] A. Newell, Z. Huang, and J. Deng, "Associative embedding: End-toend learning for joint detection and grouping," in Proc. Adv. Neural Inf. Process. Syst., 2017, pp. 2277-2287.

[64] X. Zhou, J. Zhuo, and P. Krahenbuhl, "Bottom-up object detection by grouping extreme and center points," in Proc. IEEE Conf. Comput. Vis. Pattern Recognit. (CVPR), Jun. 2019, pp. 850-859.
[65] Z. Dong, G. Li, Y. Liao, F. Wang, P. Ren, and C. Qian, "CentripetalNet: Pursuing high-quality keypoint pairs for object detection," 2020, arXiv:2003.09119. [Online]. Available: http://arxiv.org/abs/ 2003.09119

[66] J. Huang et al., "Speed/accuracy trade-offs for modern convolutional object detectors," in Proc. IEEE CVPR, vol. 4, Jul. 2017, pp. 7310-7311.

[67] A. Shrivastava, R. Sukthankar, J. Malik, and A. Gupta, "Beyond skip connections: Top-down modulation for object detection," 2016, arXiv:1612.06851. [Online]. Available: http://arxiv.org/abs/1612.06851

[68] H. Hu, J. Gu, Z. Zhang, J. Dai, and Y. Wei, "Relation networks for object detection," in Proc. Comput. Vis. Pattern Recognit. (CVPR), vol. 2, Jun. 2018, pp. 3588-3597.

[69] J. Long, E. Shelhamer, and T. Darrell, "Fully convolutional networks for semantic segmentation," in Proc. IEEE Conf. Comput. Vis. Pattern Recognit. (CVPR), Jun. 2015, pp. 3431-3440.

[70] A. Paszke et al., "Pytorch: An imperative style, high-performance deep learning library," in Proc. Adv. Neural Inf. Process. Syst., 2019, pp. 8026-8037.

[71] H.-Y. Chen et al., "TensorFlow: A system for large-scale machine learning," in Proc. OSDI, vol. 16, 2016, pp. 265-283.

[72] S. Gidaris and N. Komodakis, "Object detection via a multi-region and semantic segmentation-aware CNN model," in Proc. IEEE Int. Conf. Comput. Vis., Dec. 2015, pp. 1134-1142.

[73] N. Bodla, B. Singh, R. Chellappa, and L. S. Davis, "Soft-NMSImproving object detection with one line of code," in Proc. IEEE Int. Conf. Comput. Vis., Oct. 2017, pp. 5561-5569.

[74] Y. Chen, C. Han, N. Wang, and Z. Zhang, "Revisiting feature alignment for one-stage object detection," 2019, arXiv:1908.01570. [Online]. Available: http://arxiv.org/abs/1908.01570

[75] X. Zhou, J. Zhuo, and P. Krähenbühl, "Bottom-up object detection by grouping extreme and center points," 2019, arXiv:1901.08043. [Online]. Available: http://arxiv.org/abs/1901.08043

[76] M. Everingham, L. Van Gool, C. K. I. Williams, J. Winn, and A. Zisserman, "The Pascal visual object classes (VOC) challenge," Int. J. Comput. Vis., vol. 88, no. 2, pp. 303-338, Jun. 2010. 